Pacific Journal of Mathematics

ON A CLASSICAL THEOREM OF NOETHER IN IDEAL 


\title{
ON A CLASSICAL THEOREM OF NOETHER \\ IN IDEAL THEORY
}

\author{
ROBERT W. GILmer
}

A classical result in the ideal theory of commutative rings is that an integral domain $D$ with unit is a Dedekind domain if and only if $D$ is noetherian, of dimension less than two, and integrally closed. [8;275]. The statement of this theorem is due essentially to Noether [6; 53], though the present statement is a refined version of Noether's theorem. (See Cohen [1;32] for the historical development of the theorem above.) Noether did not, in fact, require that the domain $D$ contain a unit element. By imposing greater restrictions on the prime ideal factorization of each ideal, she showed that $D$ must contain a unit element.

This paper considers an integral domain $J$ with Property $C$ : Every ideal of $J$ may be expressed as a product of prime ideals.

In particular, it is shown that an integral domain $J$ with property $C$ need not contain a unit element. However, factorization of an ideal as a product of prime ideals is unique and $J$ is noetherian, of dimension less than two, and integrally closed. ${ }^{1}$ A domain without unit having these three properties need not have property $C$. If $J$ does not contain a unit element, $J$ is the maximal ideal of a discrete valuation ring $V$ of rank one such that $V$ is generated over $J$ by the unit element $e$, and conversely. The structure of all such valuation rings $V$ is known. [4; 62].

If $J$ is an integral domain with quotient field $k$, then $J^{*}$ will denote the subring of $k$ generated by $J$ and the unit element $e$ of $k$. We will assume that all domains considered contain more than one element.

If $D$ is an integral domain, not necessarily containing a unit, and if $k$ is the quotient field of $D$, the definitions of fractionary ideals of $D$, of sums, products and quotients of fractionary ideals, and of the fractionary ideal $\left(u_{1}, u_{2}, \cdots, u_{t}\right)$ of $D$ generated by finitely many elements $u_{1}, u_{2}, \cdots, u_{t}$ of $k$, are generalized in the obvious ways. In particular, $D^{*}$ is a fractionary ideal of $D$ and if $\mathscr{S}$ is the collection of all nonzero fractionary ideals of $D, \mathscr{S}$ is an abelian semigroup under multiplication with unit element $D^{*}$. A fractionary ideal $F$ of

Received January 22, 1963. This research was supported by the Office of Naval Research under contract number NONR G 00099-62.

1 A domain $D$ with quotient field $k$ is integrally closed if $D$ contains every element $x$ of $k$ with the following property: There exist elements $d_{0}, d_{1}, \cdots, d_{n}$ of $D$ such that $x^{n+1}+d_{n} x^{n}+\cdots+d_{1} x+d_{0}=0$. 
$D$ is said to be invertible if $F$ has an inverse when considered as an element of $\mathscr{S}$. A nonzero principal fractionary ideal is invertible and $(d)^{-1}=(1 / d)$. A product of fractionary ideals is invertible if and only if each of the factors is invertible. [3; 271].

The following two lemmas may be proved by making minor changes in the usual proofs given in the case of a domain with unit. $[8 ; 272-273]$. While the proof of Theorem 1 is definitely a modification of the usual proof for a domain with unit, the author feels enough difficulties arise to prove Theorem 1 here.

Lemma 1. If $A$ is an invertible fractional ideal of the integral domain $D$, then $A^{-1}=D^{*}$ : A. Further, $A$ has a finite module basis over $D$.

Lemma 2. Suppose $A$ is a proper ideal of the domain $D$ such that $A$ may be expressed as a product of invertible prime ideals of $D$. This representation is unique if $D \subset D^{*}$, or unique to within factors of $D$ if $D=D^{*}$.

Henceforth in this paper, $J$ will denote an integral domain without unit such that $J$ has property $C$.

THeOREM 1. Every nonzero proper prime ideal of $J$ is invertible and maximal.

Suppose first that there exists a nonzero proper invertible prime ideal $P$ of $J$ such that $P$ is not maximal. We chose $a$ such that $P \subset P+($ a $) \subset J$. We express $P+(a)$ and $P+\left(a^{2}\right)$ as products of prime ideals: $P+(a)=J^{k} P_{1} \cdots P_{r}, P+\left(a^{2}\right)=J^{t} Q_{1} \cdots Q_{s}$ where each $P_{i}$ and each $Q_{j}$ is a proper ideal of $J$. In $\bar{J}=J / P$ we have: $(\bar{a})=\bar{J}^{k} \bar{P}_{1} \cdots \bar{P}_{r}$, $(\bar{a})^{2}=\bar{J}^{t} \bar{Q}_{1} \cdots \bar{Q}_{s}$. By Lemma $2, s=2 r$ and by proper labeling $P_{i}=$ $Q_{2 i-1}=Q_{2 i}$. If $\bar{J}$ does not contain a unit element, then Lemma 2 implies also that $t=2 k$ so that $P+\left(a^{2}\right)=[P+(a)]^{2}$. If $\bar{J}$ contains a unit, then $(\bar{a})=\bar{J}^{k} \bar{P}_{1} \cdots \bar{P}_{r}$ so that $r$ is positive and $(\bar{a})=\overline{P_{1} \cdots P_{r}}$. Similarly, $(\bar{a})^{2}=\overline{Q_{1} \cdots Q_{s}}$. Therefore $[P+(a)]^{2}=P_{1}^{2} \cdots P_{r}^{2}=P+\left(a^{2}\right)$. For either case, therefore, $P+\left(a^{2}\right)=[P+(a)]^{2}$. The remainder of the proof of the theorem is the same as the proof appearing in $[8 ; 273]$.

THEOREM 2. $J$ is a noetherian domain.

We first show that $J$ is finitely generated. Thus if $J$ contains a proper nonzero prime ideal $P$, then $P=\left(p_{1}, \cdots, p_{s}\right)$ is maximal and 
finitely generated by Theorem 1 and Lemma 1 . Therefore if $d \in J$, $d \notin P$, then $J=\left(p_{1}, \cdots, p_{s}, d\right)$. If (0) is the only prorer prime ideal of $J$, then given $d \in J, d \neq 0,(d)=J^{k}$ for scme integer $k \geqq 1$. Then $J$ is invertible, and hence finitely generated.

It follows that every prime ideal of $J$ is finitely generated. Since $J$ has property $C$, every ideal of $J$ is finitely generated.

THeOREM 3. Every nonzero ideal of $J$ is a power of $J$ and, in fact, $J$ is a principal ideal domain.

Since $J$ is noetherian and $J \subset J^{*}, J^{2} \subset J$. [5; 172-73]. We choose $x \in J, x \notin J^{2}$. Because $J$ has property $C,(x)$ is prime. We shall show that $(x)=J$. We suppose that $(x) \subset J$. Because $(x)$ is invertible and $J \subset J^{*},(x) \supset(x) J \supset\left(x^{2}\right)$. If $A$ is any ideal such that $(x) \supset A \supset\left(x^{2}\right)$ and if $P$ is a prime factor of $A$, then $P \supseteqq(x)$ so that $P=(x)$ or $P=$ $J$. Because $(x) \supset A \supset\left(x^{2}\right), A=(x) J^{k}$ for some $k \geqq 1$. But $x \notin J^{2}$ so that $x^{2} \notin(x) J^{k}$ for $k \geqq 2$. Therefore $k=1$ and $(x) J$ is the unique ideal properly between $(x)$ and $\left(x^{2}\right)$.

We next show that $\left(x^{2}\right)$ is a primary ideal. Thus if $a, b \in J$, $a b \in\left(x^{2}\right)$, and $a \notin(x)$, then $b \in(x)$. Hence $\left(x^{2}\right) \subseteq\left(x^{2}, b\right) \subseteq(x)$. Now $(x)$ is maximal and prime in $J$ so that $J /(x)$ contains a unit element $\bar{u}$. Because $a \notin(x), u a \notin(x)$ so that $u a x \notin\left(x^{2}\right)$ and therefore $u x \notin\left(x^{2}, b\right)$. This means $\left(x^{2}, b\right) \nsupseteq(x) J$ so that $\left(x^{2}, b\right)=\left(x^{2}\right)$ by the preceding paragraph. Hence $b \in\left(x^{2}\right)$ and $\left(x^{2}\right)$ is primary.

Now $u a-a \in(x)$ so that $(u a-a)^{3} \in\left(x^{2}\right)$. If $z \in J$, then $z(u a-a)^{3}=$ $a^{3}(t z-z) \in\left(x^{2}\right)$ where $t$ is a fixed element of $J$ independent of $z$. Since $a^{3} \notin(x)$ and $\left(x^{2}\right)$ is primary, $t z-z \in\left(x^{2}\right)$ for each $z \in J$-i.e., $J /\left(x^{2}\right)$ contains a unit element. This means, however, that $V=(x) /\left(x^{2}\right)$ is a vector space over the field $J /(x)$. There is a one-to-one correspondence between subspaces of $V$ and ideals of $J$ between $(x)$ and $\left(x^{2}\right)$. Hence $V$ has exactly one nonzero proper subspace, which is impossible. Therefore $J=(x)$ as asserted.

If $P$ is a proper prime ideal of $J$, the argument above shows that $P \subseteq J^{2}=\left(x^{2}\right)$. This means for some ideal $A$ of $J, P=A(x)$. Since $P$ is prime, $P=A$. Now $(x)=J \subset J^{*}$ so that $P$ is not invertible and thus $P=(0)$. Hence $J$ is the only nonzero prime ideal of $J$. Therefore if $A$ is a nonzero ideal of $J, A=J^{k}=\left(x^{k}\right)$ for some positive integer $k$.

A ring $R$ with at most two prime ideals is called a primary ring. Theorem 3 shows that $J$ is a primary domain. The author has investigated primary rings in [3].

THEOREM 4. $J^{*}$ is a discrete valuation ring of rank one. Conversely if $D$ is a discrete valuation ring of rank one with maximal 
ideal $M$ and if $D=M^{*}$, then $M$ is a domain without unit having property $C$.

The proof will use the following.

Lemma 3. Suppose $S$ is a ring with unit $e$ and that $R$ is a subring of $S$ such that $S$ is generated by $R$ and $e$. $A$ subset of $R$ is an ideal of $S$ if and only if it is an ideal of $R$. $S$ is noetherian if and only if $R$ is noetherian.

For the proof of the lemma, see [3].

To prove the theorem, we let $\xi \in k$, the quotient field of $J^{*}$. For some elements $a$ and $b$ of $J, \xi=a / b$. By Theorem 3 the ideals $(a)$ and $(b)$ of $J$ compare-i.e. $a / b \in J^{*}$ or $b / a \in J^{*}$. Therefore, $J^{*}$ is a valuation ring. Because $J^{*}$ is noetherian, $J^{*}$ is discrete and of rank one. [9; 41].

If $M$ is the maximal ideal of $J^{*}$ then $J=M^{r}$ for some $r$. Then $M^{r+1} \subset J$ implies $M^{r+1}=\left(M^{r}\right)^{s}$ for some integer $s$ so that $r+1=r s$ and $r=1-$ i.e., $J=M$. Hence $J^{*} / J$ is a field. Because $J^{*}$ is generated over $J$ by $e, J^{*} / J=Z /(p)$ for some prime integer $p$.

The proof of the converse is an immediate consequence of Lemma 3 and of the fact that a discrete valuation ring of rank one is a Dedekind domain. [8; 278].

It is possible to classify all discrete valuation rings $V$ of rank one such that $V=M^{*}$ where $M$ is the maximal ideal of $V$, for if $V$ has this property, so does the completion $\bar{V}$ of $V$. [2; 60]. If now $p$ is a fixed prime, if $\Pi$ denotes the prime field with $p$ elements, $x$ an indeterminate over $\pi$, if $V_{1}=Z_{(p)}$ and $V_{2}=(\Pi[x])_{(x)}$ then $V_{1}$ and $V_{2}$ are discrete valuation rings of rank one and with residue field $\Pi$. Further $V_{1}$ and $V_{2}$ are regular and unramified in Cohen's sense. [2; 88]. Thus $\bar{V}_{1}$ and $\bar{V}_{2}$ are so-called p-adic rings. [2; 59-60, 89]. Now $\bar{V}_{1}$ has characteristic zero (unequal characteristic case for $\bar{V}_{1}$ and its residue field) and $\bar{V}_{2}$ has characteristic $p$ (equal characteristic case). The within isomorphism, $\bar{V}_{1}$ and $\bar{V}_{2}$ are the only two $p$-adic rings of dimension one having residue class field $\Pi$. [2; 89]. Now $\bar{V}_{1}$ is simply the domain of Hensel's $p$-adic integers and $\bar{V}_{2}$ is the domain of formal power series in one indeterminate over the field II. [7; 242-243]. Finally, $\bar{V}$ is an Eisenstein extension of $\bar{V}_{1}$ or $\bar{V}_{2}$, and in case $\bar{V}$ has characteristic $p, \bar{V} \cong \bar{V}_{2}$. In short we have: If $V$ has characteristic $p$, then to within isomorphism $V$ is a ring between $V_{2}$ and $\bar{V}_{2}$. If $V$ is unramified of characteristic 0 , then $V_{1} \subseteq V \subseteq \bar{V}_{1}$. If $V$ is ramified of characteristic zero, then $V$ is isomorphic to a valuation ring contained in an Eisenstein extension of $\bar{V}_{1}$. Conversely, 
if $V$ is a ring having any of the three properties just described, $V$ is a discrete valuation ring of rank one having residue field $\Pi$. [2; 59-60].

We add the following remarks:

In the last paragraph of the proof of Theorem 2, it is not necessary to use the fact that $J$ has property $C$ to conclude $J$ is noetherian. That $J$ is noetherian follows from a theorem of Cohen $[1 ; 29]$ if all prime ideals of $J$ are finitely generated.

In the proof of Theorem 3, it is not true in general that if $D /(x)$ is a field, that the ring $D /\left(x^{2}\right)$ contains a unit element, and hence that $(x) /\left(x^{2}\right)$ is a vector space over $D /(x)$. One can take $D$ to be the ring of even integers and $x=6$.

Theorem 3 implies that $J$ is noetherian and of dimension less than two. Using Theorem 4 , it is easily seen that $J$ is integrally closed. That these three conditions do not imply that a domain $D$ has property $C$ may be seen by taking $D$ to be the domain of even integers. Theorems 3 and 4 imply a bit more than the above. They even imply that $J$ is a noetherian integrally closed primary domain. It can be shown that a noetherian integrally closed primary domain $D$ without unit is the Jacobson radical of $D^{*}$, which is a semi-local ring, and that further, $D^{*} \mid D \cong Z /\left(p_{1} p_{2} \cdots p_{k}\right)$ for some distinct primes $p_{1}, \cdots, p_{k}$. [3]. However, $D$ need not have property $C$ as can be seen by choosing $D$ as the Jacobson radical of $Z_{M}$ where $M$ consists of all integers relatively prime to 6 . An analog to the classical Noether theorem cited earlier in the case of a domain without unit, while obtainable, now seems not as desirable to the author as Theorem 4 .

\section{REFERENCES}

1. I. S. Cohen, Commutative rings with restricted minimum condition, Duke Math. J. 17 (1950), 27-42.

2. On the structure and ideal theory of complete local rings, Trans. Amer. Math Soc., 59 (1946), 54-106.

3. R. W. Gilmer, Commutative rings containing at most two prime ideals, submitted to Michigan Math. J.

4. H. Hasse and F. K. Schmidt, Die Struktur diskret bewerteter Korper, J. Reine Angew. Math., 170 (1934), 4-63.

5. S. Mori, Über Ringe, in denen die grossten primarkomponenten jedes Ideals eindeutig bestimmt sind, J. Science Hiroshima U., Ser. A, 1, (1931), 160-193.

6. E. Noether, Abstrakter aufbau der Idealtheorie in algebraischen zahl-und Funktionenkorpern, Math., Ann., 96 (1927), 26-61.

7. B. L. van der Waerden, Modern algebra, v. 1. Ungar, New York, 1949.

8. O. Zariski and P. Samuel, Commutative algebra v. 1, von Nostrand, Princeton, 1958.

9. — Commutative algebra, V. 2, Von Nostrand, Princeton, 1960. 



\title{
PACIFIC JOURNAL OF MATHEMATICS
}

\author{
EDITORS
}

\author{
Ralph S. Phillips \\ Stanford University \\ Stanford, California \\ M. G. Arsove \\ University of Washington \\ Seattle 5 , Washington
}

\author{
J. DugunduI \\ University of Southern California \\ Los Angeles 7, California
}

Lowell J. Paige

University of California

Los Angeles 24, California

\section{ASSOCIATE EDITORS}

E. F. BECKENBACH

T. M. CHERRY
D. DERRY
M. OHTSUKA

H. L. ROYDEN

E. SPANIER
E. G. STRAUS

F. WOLF

\section{SUPPORTING INSTITUTIONS}

UNIVERSITY OF BRITISH COLUMBIA CALIFORNIA INSTITUTE OF TECHNOLOGY UNIVERSITY OF CALIFORNIA MONTANA STATE UNIVERSITY UNIVERSITY OF NEVADA NEW MEXICO STATE UNIVERSITY OREGON STATE UNIVERSITY UNIVERSITY OF OREGON OSAKA UNIVERSITY UNIVERSITY OF SOUTHERN CALIFORNIA
STANFORD UNIVERSITY

UNIVERSITY OF TOKYO

UNIVERSITY OF UTAH

WASHINGTON STATE UNIVERSITY

UNIVERSITY OF WASHINGTON

AMERICAN MATHEMATICAL SOCIETY CALIFORNIA RESEARCH CORPORATION SPACE TECHNOLOGY LABORATORIES NAVAL ORDNANCE TEST STATION

Mathematical papers intended for publication in the Pacific Journal of Mathematics should be typewritten (double spaced), and the author should keep a complete copy. Manuscripts may be sent to any one of the four editors. All other communications to the editors should be addressed to the managing editor, L. J. Paige at the University of California, Los Angeles 24, California.

50 reprints per author of each article are furnished free of charge; additional copies may be obtained at cost in multiples of 50 .

The Pacific Journal of Mathematics is published quarterly, in March, June, September, and December. Effective with Volume 13 the price per volume (4 numbers) is $\$ 18.00$; single issues, $\$ 5.00$. Special price for current issues to individual faculty members of supporting institutions and to individual members of the American Mathematical Society: $\$ 8.00$ per volume; single issues \$2.50. Back numbers are available.

Subscriptions, orders for back numbers, and changes of address should be sent to Pacific Journal of Mathematics, 103 Highland Boulevard, Berkeley 8, California.

Printed at Kokusai Bunken Insatsusha (International Academic Printing Co., Ltd.), No. 6, 2-chome, Fujimi-cho, Chiyoda-ku, Tokyo, Japan.

PUBLISHED BY PACIFIC JOURNAL OF MATHEMATICS, A NON-PROFIT CORPORATION

The Supporting Institutions listed above contribute to the cost of publication of this Journal, but they are not owners or publishers and have no responsibility for its content or policies. 


\section{Pacific Journal of Mathematics}

\section{Vol. 13, No. 2 \\ April, 1963}

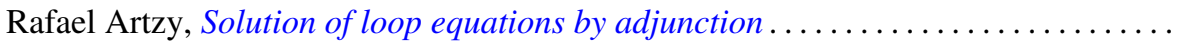

Earl Robert Berkson, A characterization of scalar type operators on reflexive

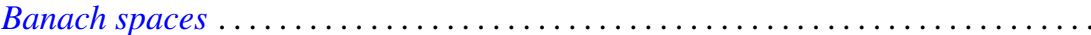

Mario Borelli, Divisorial varieties

365

Raj Chandra Bose, Strongly regular graphs, partial geometries and partially

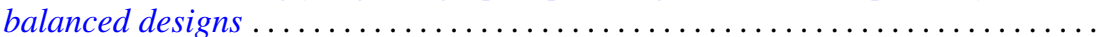

389

R. H. Bruck, Finite nets. II. Uniqueness and imbedding ............... 421

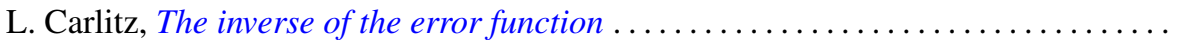

Robert Wayne Carroll, Some degenerate Cauchy problems with operator

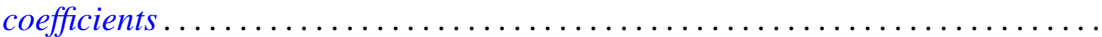

Michael P. Drazin and Emilie Virginia Haynsworth, A theorem on matrices of 0 's

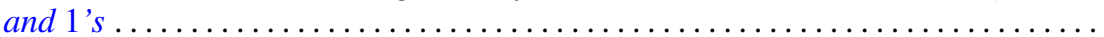

Lawrence Carl Eggan and Eugene A. Maier, On complex approximation .......... James Michael Gardner Fell, Weak containment and Kronecker products of group

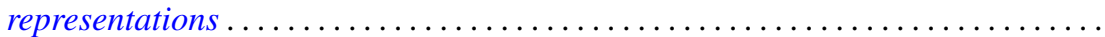

Paul Chase Fife, Schauder estimates under incomplete Hölder continuity

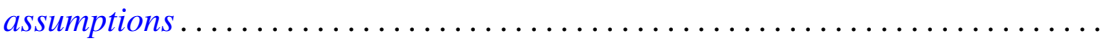

Shaul Foguel, Powers of a contraction in Hilbert space ...................

Neal Eugene Foland, The structure of the orbits and their limit sets in continuous

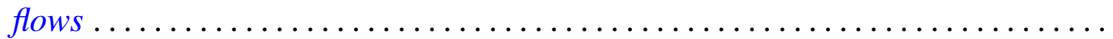

Frank John Forelli, Jr., Analytic measures . . . . . . . . . . . . . . . . . . . . . 563

Robert William Gilmer, Jr., On a classical theorem of Noether in ideal theory ....... P. R. Halmos and Jack E. McLaughlin, Partial isometries .

Albert Emerson Hurd, Maximum modulus algebras and local approximation in

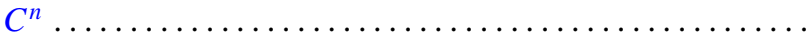

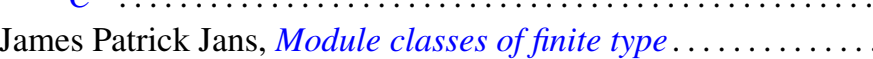

Betty Kvarda, On densities of sets of lattice points ...

H. Larcher, A geometric characterization for a class of discontinuous groups of linear fractional transformations .

John W. Moon and Leo Moser, Simple paths on polyhedra .

T. S. Motzkin and Ernst Gabor Straus, Representation of a point of a set as sum of

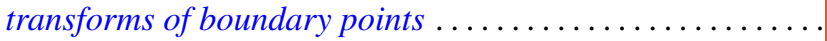

Rajakularaman Ponnuswami Pakshirajan, An analogue of Kolmogorov's three-series theorem for abstract random variables ...............

Robert Ralph Phelps, Čebyšev subspaces of finite codimension in $C(X)$...

James Dolan Reid, On subgroups of an Abelian group maximal disjoint from a given subgroup ...

William T. Reid, Riccati matrix differential equations and non-oscillation criteria for associated linear differential systems .................

Georg Johann Rieger, Some theorems on prime ideals in algebraic number fields ...

Gene Fuerst Rose and Joseph Silbert Ullian, Approximations of functions on the

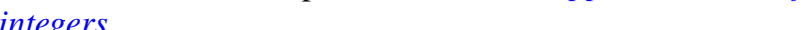

F. J. Sansone, Combinatorial functions and regressive isols . . . . . . . . . . 703

Leo Sario, On locally meromorphic functions with single-valued moduli . . . . . . . 709

Takayuki Tamura, Semigroups and their subsemigroup lattices.

Pui-kei Wong, Existence and asymptotic behavior of proper solutions of a class of second-order nonlinear differential equations . . ........... 